\title{
Restoring the Sense of Self in the Process of Recovery in Schizophrenia
}

\author{
Sonal Mathur • Poornima Bhola • Fatema Khanam • \\ Jagadisha Thirthalli
}

Received: 5 March 2014/Revised: 9 April 2014/Accepted: 10 April 2014/Published online: 25 May 2014

(C) Springer India Pvt. Ltd. 2014

\begin{abstract}
Recovery oriented services extend beyond reduction of psychiatric symptoms and focus on helping individuals reconstruct their lives in a meaningful way. This case report describes the rehabilitation interventions with a 37 year old woman with chronic schizophrenia and residual disability during a four-month inpatient stay. These comprised individual supportive psychotherapy with vocational rehabilitation, social skills training and behavioural management components. Family intervention addressed maternal criticality and over involvement. The reduction of internalized stigma and changes in the family interactions and dynamics were important for achieving therapeutic gains. The therapeutic process demonstrates how a person's selfhood can expand beyond being defined by the illness by restoring the sense of agency and generating hope. The therapeutic challenges, implications for clinical practice, rehabilitation services and future research are discussed.
\end{abstract}

Keywords Internalized stigma $\cdot$ Recovery ·

Rehabilitation $\cdot$ Schizophrenia $\cdot$ Self

\section{Introduction}

The philosophy of recovery in mental health rehabilitation [1] emphasises the consumer perspective and the need to move beyond reduction of symptoms to focus on the restoration of

S. Mathur · P. Bhola $(\bowtie) \cdot$ F. Khanam

Department of Clinical Psychology, National Institute of Mental

Health and Neuro Sciences, Bangalore 560008, India

e-mail: poornimabhola@gmail.com

J. Thirthalli

Department of Psychiatry, National Institute of Mental Health and Neuro Sciences, Bangalore, India the sense of self. The parameters of rehabilitation frameworks have expanded and shifted from the notion of recovery 'from' mental illness to meaningful recovery 'in' mental illness, within the limits of the disability [2].

There is a growing recognition that schizophrenia as an illness impacts the sense of self and social identity, apart from the disturbances in perception, cognition and functioning [3]. Studies suggest that people diagnosed with schizophrenia internalize the public stigma and discrimination related to mental illnesses [4]. Internalized stigma is also seen as contributing to low levels of self-esteem and self-efficacy, creating barriers towards a productive social and occupational life and lowering the overall quality of life [5]. Social and interpersonal processes are implicated in persons assuming a devalued 'patient role' [6], and this subjective experiences of the illness may be manifested in lowered agency, hopelessness and social withdrawal. The process of rehabilitation and recovery calls for the recognition of this 'secondary disability' and processes to help individuals reconstruct temporally coherent and unified autobiographical accounts [7]. The terrain of re-authoring one's life story after changes wrought by the illness must be integral to and not seen merely as a potential by-product of recovery [7].

The case report illustrates the manifestations of disturbances in self and personhood in an individual living with schizophrenia. It highlights the role of multi-pronged recovery-oriented inpatient rehabilitation services to address family interaction patterns, social and vocational functioning and initial steps towards restoration of the sense of self.

\section{Summary of the Client's History}

Ms. SS was a 37 year old divorced woman from middle socioeconomic status living with her widowed mother, 
with a 15 year history of markedly decreased social interaction, forgetfulness and slowness in doing all activities with an insidious, continuous and progressive course. There was family history suggestive of schizoid traits and possible psychotic symptoms in father and a psychotic illness in a second degree relative. Subsequent to father's death when Ms. SS was 8 years old, the mother had been the primary caretaker for her and her elder brother. The mother was reported to be a perfectionist and strict disciplinarian. Premorbidly, the client was introverted with few friends. Her personal history indicated average scholastic performance with later failures during graduate studies and an unstable employment pattern, with brief periods as a nursery school teacher. Her arranged marriage ended in divorce after three months, with reported difficulties in coping with demands of running her own household and suspicions about husband's fidelity. The mother indicated a possibility that the husband had indeed been involved in an extra-marital relationship but was unable to confirm this. Past history noted periods with irrelevant talk, decreased sleep and crying. Previous consultation records indicated a diagnosis of Schizophrenia and irregular treatment with inadequate trials of antipsychotics and antidepressants. The mental status examination at admission revealed no psychotic symptoms, but she had decreased tone, tempo and volume of speech and memory difficulties. Ward observations indicated apathy and poor motivation along with criticality and over-involvement in mother's interactions with the client.

\section{Psychological Assessment and Rehabilitation Plan}

Psychological assessment included comprehensive evaluation of memory [8] and neuropsychological functioning [9].

The findings suggested bi-frontal left temporal involvement with deficits in visual memory, sustained attention, speed of information processing, response inhibition, planning and set shifting. There was low-average functioning in working memory. Dysfunctional connectivity in the frontotemporal regions has been implicated in schizophrenia [10].

Assessment with projective tests was planned in view of the initial inadequate history and documentation of past symptoms and the absence of any positive psychotic symptoms on the mental status examination. Findings indicated adequate reality orientation, low ego strength, poor self-concept and self-acceptance. Findings also indicated vulnerability to emotional impact from the external environment and a withdrawn and passive stance.

Behavioural observations noted slowness in task performance, awareness and anxiety about errors and frequent reassurance seeking. A diagnosis of Schizophrenia with residual disability guided the intervention plan. The intersections of neuro-cognitive vulnerability and psychosocial stress factors like interpersonal criticism [11] were seen as jointly contributing to the dysfunction. The rehabilitation plan encompassed multiple perspectives; individual supportive psychotherapy for the client with vocational rehabilitation, social skills training and behavioural management components and family intervention during a four-month inpatient stay. She was receiving clozapine $150 \mathrm{mg} /$ day, escitalopram $20 \mathrm{mg} /$ day and donepezil $10 \mathrm{mg} /$ day from a previous psychiatrist. While the latter two medications were continued at the same dose, clozapine was tapered and stopped; iloperidone at $6 \mathrm{mg} /$ day was prescribed instead.

\section{Initial Phase: The "Why Try" Effect}

The initial phase aimed to provide a safe and validating therapeutic environment. A biopsychosocial model was used to provide psychoeducation about the illness and pathways to recovery. This included 6 individual sessions and initiation of joint mother-daughter sessions by separate therapists. This phase was dominated by barriers and hesitation about engaging in any independent or new activities. The client's voice was barely heard, with primarily “I don't know' answers and mother acting as the spokesperson. The client tended to agree with mother's descriptions of difficulties in performing any task competently and independently, without any emotional expression and limited eye contact. The therapist encountered difficulties in facilitating the client's expression of her interests, needs, difficulties or aspirations and in estimating the client's potential.

Initial efforts at working towards behavioural activation were met with repeated expressions of uncertainty and inadequacy. Both client and mother used the term "absentminded" to explain her limited potential and difficulties in engagement towards even minimal change. This "why try" effect has been described in persons with a mental illness [12]; comprising internalization of devaluation, lowered self-esteem and efficacy and inadequate efforts towards goal attainment.

The client's negative expressions seemed out of proportion to her cognitive, social and instrumental deficits. The therapist began to realize the extent of scaffolding and validation that would be required in the therapeutic space. Explorations in the initial phase revealed that, although the client constantly undermined her abilities, she had completed a basic tailoring course in the past. The vocational rehabilitation plan included a referral for graded individualized training in the computer and tailoring sections. Research has indicated the positive impact of vocational rehabilitation on internalized stigma and self-esteem [13].

The client was also engaged in cooking daily meals to enhance independent activities of daily living. During this 
phase, the constant instructions, supervision and evaluative comments from the mother posed additional barriers. Another therapist stepped into conduct individual sessions with the mother to address the negative expressed emotions [14] and change her role from supervisor to observer. The resistance to the goals of client autonomy was prominent during the initial phase.

\section{Middle Phase: Shifts in Empowerment, Expressed Emotions and Enmeshment}

This phase involved 24 individual therapy sessions with the client, 6 individual sessions with the mother and 18 joint sessions with the client-mother dyad held concurrently. This phase began with planned separation of mother and client for part of the day while she was engaged in activities of independent living and vocational training. Joint sessions were held to negotiate the tasks which client could do without maternal evaluation. The mother was noticed to frequently interrupt and speak for the patient, emphasise the lack of positive change and the need for her own continued presence and involvement.

Individual sessions with the mother validated her concerns for her daughter's wellbeing but continued to address the areas of appropriate communication and reinforcement strategies.

On one hand, the client appeared well-integrated, with a tendency to make more errors in the mother's presence. However, she also demonstrated difficulties organising, planning and remembering tasks in the therapy sessions and learning skills in the vocational sections. This introduced uncertainties regarding pacing of the tasks and goals of therapy as well as in recognising the initial gains made by the client. During sessions with the client, the potential utility of memory aids, like a 'to-do' list or timetable, was discussed.

The therapist also provided positive feedback about the client's involvement, and the gradual increase in speed and learning during cooking, computer, tailoring and yoga activities.

During sessions, there was little spontaneous communication and also limited social interactions with others outside of the therapy room. Gradually, the client reported anxieties about being rejected or ridiculed by others. Attempts to socialise with relatives, neighbours, employers or co-workers had inevitably ended in her being called absent-minded or similar labels related to her mental illness. Initially, these incidents were mentioned briefly, without any associated emotion. In a significant therapeutic moment, the client was tearful and expressed her pain at being regarded as not "goodenough" by mother, brother and others in society. This unexpected expression of vulnerability and the emotional impact of being labelled initially caught the therapist unprepared and unsure about how to comfort the client. As observed by Lysaker \& Lysaker [15], persons with schizophrenia who display flat affect, may in fact, experience an intense emotional reactivity that contradicts their lack of overt affective expression. The critical incident brought the therapist's underlying assumptions about the client's apparent dispassionate stance into sharp focus. This pivotal moment helped the therapist to empathise with the client and increase the supportive and validating stance in later phases of therapy. The client's expression of vulnerability in the safe space of therapy also served as feedback to the therapist that a therapeutic relationship had been built even through the sometimes frustratingly slow pace of change. The unexpected emotional expression opened the doors to therapist reflections on aspects of client engagement and the unfolding of therapy processes over time; and perhaps would not have been experienced with the same depth and intensity in its absence.

This single incident had a powerful impact in terms of a better understanding of the client's lived experience, strengthened the therapeutic relationship and underlined the importance of validation, trust and unconditional positive regard in the therapeutic holding environment. Clearly, this demonstrated the experience of internalized stigma, which had a possible role in impeding her recovery. The therapist became alert to the fact that the client used the term absent-minded to describe herself each day, on several occasions and this was incorporated into discussions during sessions. According to Roe \& Davidson [7], clients need to separate their 'healthy' self from their 'ill' self. Therapeutic processes focused on engagement in positive conversations to facilitate client's descriptions of self using positive adjectives (e.g. helpful, creative). The client's dependence on the therapist for guidance and answers was de-emphasised and her own problem-solving skills were bolstered. Concurrently, there was use of graded exposure tasks and role-play scenarios to reduce social anxiety and target select social skill deficits.

As therapy progressed, there was a graded increase in task difficulty level and degree of independent functioning. These included setting up a personal email account, searching for recipes on the internet, shopping and bargaining for groceries and vegetables, participation in interactive ward functions and activities and initiating conversations with others. The client's assets in terms of a gradual increase in motivation and efforts towards individuation became evident. When she received appreciation for these gains, the client expressed relief and happiness and stopped referring to herself as absent-minded after 17 individual sessions. When she was given feedback about this, client initially started calling herself absent-minded again, but was reminded by the therapist of the other positive qualities that describe her. 
Therapeutic efforts at reducing expressed emotions and enmeshment had yielded limited and fluctuating gains and led to feelings of frustration among the treating team. The therapist recognised feelings of anger towards the mother and overprotection towards the client, with the accompanying risk of infantilizing her in therapy. The need to have empathy for the mother, and the importance of a gradual balance between autonomy and dependence in this long enmeshed relationship were also examined by the therapist. The mother was gradually encouraged to participate as a co-therapist, with more realistic expectations. The frequency of instructions and negative remarks reduced and mother began to appreciate some of the changes. This phase ended with the mother expressing that she now does not wish for her daughter to lead the exactly the kind of life she had envisioned for her, but still hopes for a little more change.

\section{Termination Phase: Consolidating Gains}

The last eight sessions (four individual and four family $\mathrm{s}$ sessions) aimed at consolidating the gains with respect to the mother-daughter relationship and the mother's role as a therapeutic agent after discharge and making specific plans for the immediate future.

Towards the termination of therapy, improvements noted were in client's positive self-statements, improved eye contact, use of "I" terminology, increased initiation of conversations, commitment towards select household responsibilities and engagement in decisions about future employment plans. During the final joint session with client, brother and mother, the client stepped into stop the brother from answering for her, and spoke of her increased confidence, hope for the future and her short-term and long-term plans.

A local referral was made for future therapy and a fourmonth telephonic follow-up indicated that the client was managing some chores at home independently and was employed for secretarial work in an office.

\section{Discussion and Reflections}

This multi-pronged therapeutic approach had goals that extended beyond reducing symptoms to rebuilding a sense of agency and a more functional life. The intervention process revealed that the internalized stigma about her illness [16] had continually been reinforced and contributed to social withdrawal and social skills deficits. The importance of micro gains, need for continued prolonged therapy sessions, and unconditional positive regard for the client's emergent sense of self-esteem and self-efficacy are highlighted in this case report. This case cautions against preconceived notions about clients' strengths and vulnerabilities without adequate assessment and sustained interaction. Systematic case studies would benefit from planned pre and post assessments in relevant areas; for example, neuropsychological functioning, self-esteem and social cognition in this case. In working with clients with a severe mental illness and residual deficits, therapists must be alert to micro gains and not await significant events or large changes. The importance of supervisory discussions was highlighted as the individual therapist often found herself joining or aligning with either the client or the mother. The multi-disciplinary approach to case management including medication, intensive inputs using varied therapy modalities and techniques and effective communication between members of the treating team helped in integrating various aspects of the recovery-oriented rehabilitation approach.

An illness like schizophrenia affects not only the client but also the family. With the family playing a central role in treatment and rehabilitation in countries like India, addressing dysfunctional interactional patterns is a priority [17]. The intertwined relationship between familial expressed emotions and the individual's internalized stigma is demonstrated in this case report. Future therapeutic goals may need to explore the mother-daughter relationship further, along with the dynamics of the larger family system in order to support the changes.

The therapeutic work represents nascent beginnings in restoring the client's sense of self and many emotional issues and experiences may have remained unaddressed. While the emergent changes were encouraging, the client's premorbid vulnerabilities, long course of the illness and the neuropsychological deficits would moderate outcomes in the long-term. This calls for realistic expectations and goalsetting from the family members and the treating team. Continued therapeutic inputs, while balancing the issues of individuation and dependency, would perhaps play a critical role in consolidating changes in self and functioning.

This case report has various implications for clinical practice. Clinicians need to be sensitive to the intersections between self, identity and the illness. What is the extent to which disruptions in the self are adversely impacting therapeutic outcome and recovery processes? Is the person (and family) engulfed in the "patient role", unable to make the distinction between "being schizophrenic" and "having schizophrenia"? The therapeutic space needs to recognise these patterns and specifically address restoration of the issues of hope, agency and self-determination in comprehensive interventions for individuals with a mental illness. The role of and indications for narrative approaches to therapy $[7,18]$ needs to be examined.

Few research implications can be derived from this case report. With evidence of cultural variations in impact of 
illness on self-esteem, internalized stigma, sense of agency and empowerment [19] this presents a promising avenue for future studies. Future research studies can add to the limited knowledge about the impact of 'illness identity' [20] and disruptions in the sense of self on recovery processes in severe mental illnesses. Efforts to explore such lived experiences must recognise that 'double-blind is not the only way to see' [18] and include qualitative methodologies as well.

The case report illustrates the need to identify strengths within the client and context and use a multi-pronged approach while specifically looking at issues of the 'self' in the recovery process from a severe mental illness like schizophrenia.

\section{References}

1. Barton R. The rehabilitation-recovery paradigm: a statement of philosophy for a public mental health system. Psychiatr Rehabil Skills. 2002;2(2):171-87.

2. Davidson L, Roe D. Recovery from versus recovery in serious mental illness: one strategy for lessening confusion plaguing recovery. J Ment Health. 2007;16(4):459-70.

3. Fabrega HJ. The self and schizophrenia: a cultural perspective. Schizophr Bull. 1989;15(2):277-90.

4. Livingston JD, Boyd JE. Correlates and consequences of internalized stigma for people with mental illness: a systematic review and meta-analysis. Soc Sci Med. 2010;71:2150-61.

5. Mittal D, Sullivan G, Chekuri L, Allee E, Corrigan PW. Empirical studies of self-stigma reduction strategies: A critical review of the literature. Psychiatr Serv. 2012. doi:10.1176/appi.ps. 201100459.

6. Estroff S. Self, identity, and subjective experiences of schizophrenia: in search of the subject. Schizophr Bull. 1989;15:189-96.

7. Roe D, Davidson L. Self and narrative in schizophrenia: time to author a new story. J Med. Ethics. 2005;31:89-94.
8. Gurappa PD. Wechsler memory scale III. Bangalore: Pearson Education; 2009.

9. Rao SL, Subbakrishna DK, Gopukumar K. NIMHANS neuropsychological battery. Bangalore: National Institute of Mental Health and Neuro Sciences; 2009.

10. John JP. Fronto-temporal dysfunction in schizophrenia: a selective review. Indian J Psychiatry. 2009;51(3):180-90.

11. Rosenfarb IS, Nuechterlein KH, Goldstein MJ, Subotnik KL. Neurocognitive vulnerability, interpersonal criticism, and the emergence of unusual thinking by schizophrenic patients during family transactions. Arch Gen Psychiatry. 2000;57:1174-9.

12. Corrigan P, Larson JE, Rusch N. Self-stigma and the 'why try' effect on life goals and evidence- based practice. World Psychiatry. 2009;8:75-81.

13. Lysaker PH, Roe D, Ringer J, Gilmore EM, Yanos PT. Change in self-stigma among persons with schizophrenia enrolled in rehabilitation: associations with self-esteem and positive and emotional discomfort symptoms. Psychol Serv. 2012;9(3):240-7. doi:10.1037/a0027740.

14. Amaresha AC, Venkatasubramanium G. Expressed emotion in schizophrenia: an overview. Indian $\mathbf{J}$ Psychol Med. 2012;34:12-20.

15. Lysaker PH, Lysaker JT. Narrative structure in psychosis: schizophrenia and disruptions in the dialogical self. Theory Psychol. 2002;12:207-20.

16. Lysaker PH, Roe D, Yanos PT. Toward understanding the insight paradox: internalized stigma moderates the association between insight and social functioning, hope and self-esteem among people with schizophrenia spectrum disorders. Schizophr Bull. 2007;33:192-9.

17. Sawant NS, Jethwani KS. Understanding family functioning and social support in unremitting schizophrenia: a study in India. Indian J Psychiatry. 2010;52:145-9.

18. Roberts GA. Narrative and severe mental illness: what place do stories have in an evidence-based world? Adv Psychiatr Treat. 2000;6:432-41.

19. Krajewski C, Burazeki G, Brand H. Self-stigma, perceived discrimination and empowerment among people with a mental illness in six countries. Psychiatr Res. 2013;210(3):1136-46.

20. Yanos PT, Roe D, Lysaker PH. The impact of illness identity on recovery from severe mental illness. Am J Psychiatr Rehabil. 2010;13(2):73-93. 